\title{
DIAGNÓSTICO Y TRATAMIENTO DE LA URETRITIS CRÓNICA EN LA SANIDAD MILITAR CON EL CAMBIO DE SIGLO (XIX-XX)
}

\author{
Fernando Martín-Laborda y Bergasa y Doroteo Lozano Lozano.
}

Clínica La Luz. Madrid. España.

Resumen.- OBJETIVO: Podemos afirmar que en los Hospitales Militares, instituciones pioneras dentro de la organización sanitaria del país, aproximadamente un $25 \%$ de la patología durante la segunda mitad del siglo XIX, correspondería a las Enfermedades Venéreas, existiendo salas dedicadas a este tipo de afecciones que inicialmente se asociaban a las Enfermedades del Aparato Genito-urinario.

La "Gota Militar" o Uretritis Blenorrágica fue un padecimiento venéreo con gran incidencia y prevalencia en la era pre-antibiótica. En el presente trabajo revisaremos los métodos diagnósticos y terapéuticos realizados por los Médicos Militares Españoles durante el periodo indicado.
MÉTODOS: Se realiza una introducción, interrelacionando los conocimientos de la época con el medio en que los Médicos Militares desarrollaban su actividad profesional. Se hace referencia a las Revistas de Sanidad Militar, vehículo de las inquietudes y demostración del nivel de conocimiento teórico y práctico que les distinguía.

En base a sus Hojas de Servicio y a sus publicaciones, hacemos mención de los Urólogos originarios de la Sanidad Militar que más interés demostraron en el campo de la patología de la uretra, comentando la metodología que utilizaban en el diagnóstico y tratamiento de la Uretritis Blenorrágica.

RESULTADOS Y CONCLUSIONES: La Sanidad Militar de finales del siglo XVIII y principios del XIX reunió a un grupo de profesionales que demostraron ingenio y perseverancia en el tratamiento de la "Gota Militar".

Conocer los conceptos en el diagnóstico y manejo de las uretritis, mejora nuestra perspectiva en el conocimiento de estas patologías y nos confirma en la valoración de los avances de que hoy disponemos, gracias a la suma de esfuerzos de nuestros predecesores.

Palabras clave: Gota Militar. Uretritis Blenorrágica. Blenorrea. Sanidad Militar. Historia de la Urología Militar.

Summary.- OBJECTIVES: We can say that in military hospitals, pioneer institutions in health-care in our country, approximately $25 \%$ of the pathology over the second half of the 19th century would correspond to sexually transmitted diseases (STD,I with hospital wards dedicated to these diseases, initially associated with genitourinary diseases. The "military drip" or blennorrhagic ure- 
thritis was a venereal disease with great incidence and prevalence in the pre-antibiotic era. This article reviews the diagnostic and therapeutic methods employed by Spanish military doctors during such period.

METHODS: In the introduction we state the relationship between the knowledge of the time and the environment in which military doctors developed their professional activity. We make reference to military health-care journals, vehicle for their worries and demonstration of the level of theoretical and practical knowledge they were distinguished for. Based on their service records and publications we refer the urologists from the military health-care system that showed a greater interest in the field of urethral diseases, talking about the methodology they used for diagnosis and treatment of blennorrhagic urethritis.

RESULTS AND CONCLUSIONS: Military health-care at the end of 18th century and the beginning of the 19th century joined a group of professionals who demonstrated talent and perseverance in the treatment of "military drip".

To know the concepts for the diagnosis and treatment of urethritis improves our perspective in the knowledge of these pathologies, and confirms us in the evaluation of the advances available for us today, thanks to the addition of efforts of our predecessors.

Keywords: Military drip. Blennorrhagic urethritis. Blennorrhea. Military health-care. History of military Urology.

\section{INTRODUCCIÓN}

Desde los comienzos del siglo XIX al iniciarse el "Periodo Contemporáneo" que culminaría en los años 1914-1918, la Medicina sufre una profunda transformación. La Medicina Práctica inmersa en los importantes cambios conceptuales y de análisis cambiará de manera radical, no solo en el diagnóstico y la interpretación de la clínica sino también en sus métodos terapéuticos. Al establecerse los comienzos del Método Anatomo-clínico, la lesión es la clave del diagnóstico y a su vez es el fundamento de la clínica. Hasta entonces, la lesión había acompañado al síntoma. El eje de la Medicina Contemporánea sería conocer la lesión que justificaría luego la interpretación de todas las manifestaciones clínicas (1).

Entre los médicos de la llamada Nueva Escuela Vienesa la máxima figura sería Karl von Rokitansky (1804-1878) que con su extraordinario "Tratado de Anatomía patológica" construiría la base del Método
Anatomoclínico. Fue Bichat (1771-1802) quien formuló los principios del mismo al proponer una nosología con base anatomopatológica, más tarde Corvisart (1775-1821) y Laënnec serían los encargados de la elaboración del Método Anatomoclínico (2).

En este marco es en el que haremos referencia a la interpretación, conocimiento y tratamientos que los Médicos Militares realizaron en la comúnmente llamada "Gota Militar". Así el diagnóstico que en un principio fue sintomático y puramente empírico, pasaría a ser analítico con la ayuda de los modernos medios exploratorios endoscópicos gracias a la uretroscopia y terapéuticos al introducirse el concepto de "asepsia", enunciado por la doctrina de Lister y popularizado en nuestro país por el cirujano catalán Salvador Cardenal en su "Manual" (1878), que sería reeditado en varias ocasiones (3).

Con el nacimiento de la anestesia clorofórmica en 1847 y el desarrollo progresivo de las técnicas de la antisepsia se inicia el desarrollo de la especialización en las diversas ramas de la Cirugía. Estas novedades, junto al instrumental quirúrgico necesario llegan al Hospital Militar de Madrid mediada la década de los sesenta, difundidas por los Cirujanos Militares que disfrutaban de la "Licencia Real" para asistir a Exposiciones y Congresos Internacionales de Ciencias Médicas. Se sabe que desde principios de 1860 ya hubo en el Hospital Militar salas especializadas solamente en determinadas patologías (Clínicas) de Oftalmología, Medicina Interna y Cirugía General (4).

Durante estos primeros años existía un gran estimulo en los profesionales que pertenecen a Sanidad Militar, favorecido por la convivencia y la comunicación entre los que estaban destinados en el Hospital Militar de Madrid, influidos por las tendencias internacionales (Figura 1). Este ambiente facilitó el nacimiento de diversas revistas y publicaciones periódicas que se irán solapando en el tiempo.

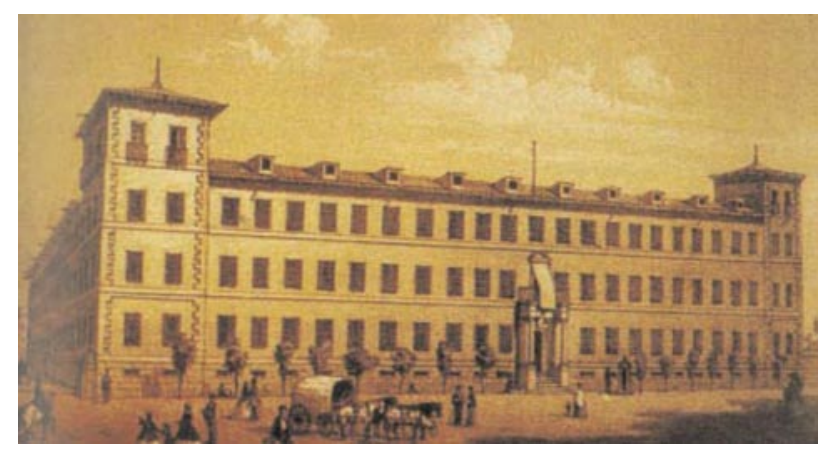

FIGURA 1. Hospital Militar de Madrid ( 1841-1899). Antiguo Seminario de Nobles. 


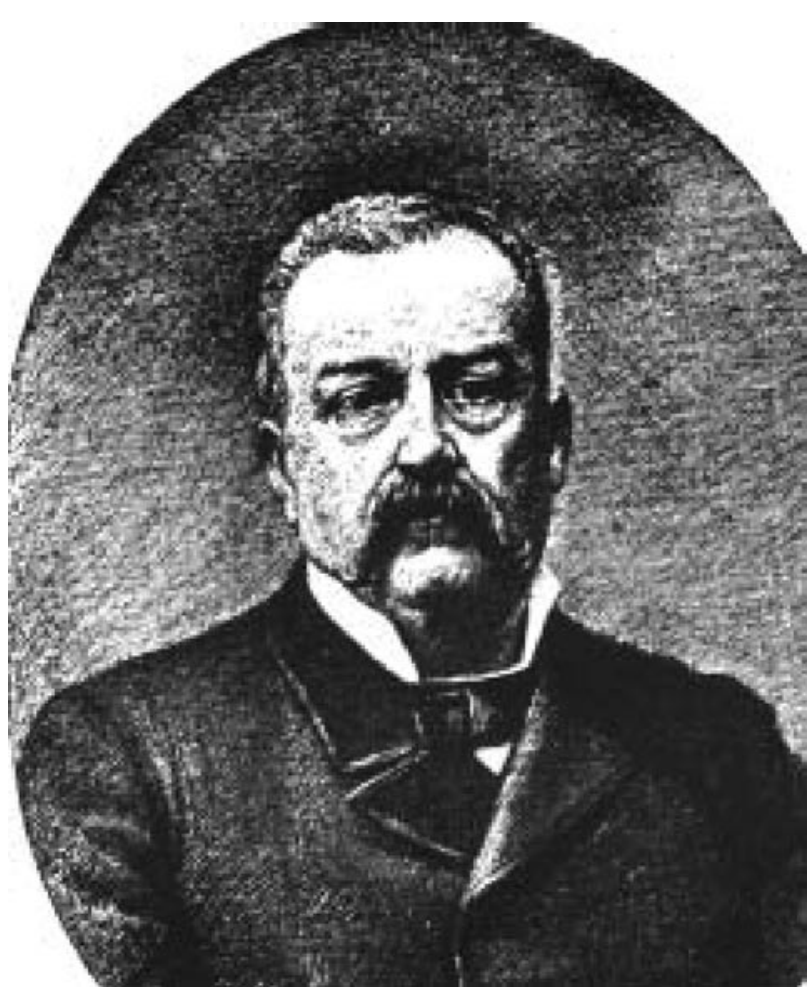

FIGURA 2. El Dr. Enrique Suender Rodríguez (1829-1897).

La "Biblioteca Médico-quirúrgica Castrense Española" de aparición bimensual y corta vida se editó desde mayo de 1851 hasta agosto de 1852. En abril de 1855 se autorizó la edición el "Boletín Oficial de Sanidad Militar" que se publicó desde julio de este año hasta finales de 1864. En 1858 Fernández y Fernández Losada fundó el "Memorial de Sanidad del Ejército y la Armada" que se creaba con la intención de recopilar y publicar trabajos sanitarios sin el carácter oficial que tenía el Boletín, esta publicación tendría una supervivencia apenas superior a un lustro y dejo de existir al coincidir con la guerra de Marruecos (5).

En 1864 se creó la "Revista de Sanidad Militar Española y Extranjera" que en 1867 cambiaría el nombre por el de "Revista General de Ciencias Médicas de Sanidad Militar" de duración efímera. En 1875 se iniciaría como publicación quincenal la "Gaceta de Sanidad Militar" que solo tuvo una vida de diez años, y por fin en 1886 los Médicos Primeros Drs. Aycart y Quintana fundarían la "Revista de Sanidad Militar" que sería remodelada más tarde en 1891 (2 $2^{a}$ época) y en 1913, (3a época) manteniendo sus características hasta 1936 (6).

A través de estas publicaciones se pueden valorar las inquietudes que desde un principio existie-

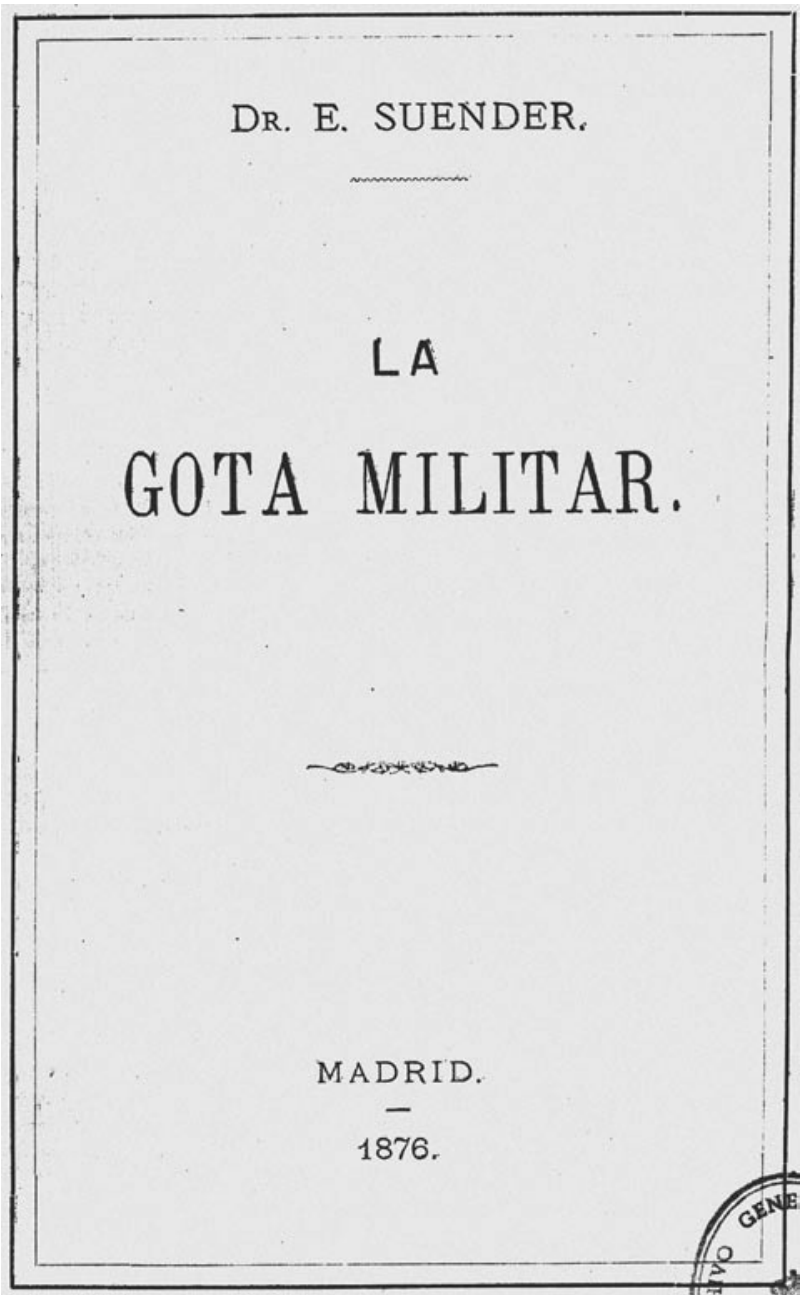

FIGURA 3. Monografía sobre "La Gota Militar" publicada en 1876 por el Dr. Suender.

ron en Sanidad Militar hacia las patologías del aparato urinario, así como la influencia e información actualizada que tenían de las novedades tanto en cuanto equipos como a técnicas desarrolladas en los países del entorno.

Tres hechos serán fundamentales para el desarrollo de la Urología como especialidad en el antiguo Hospital Militar en Madrid. En primer lugar la creación en 1856 a propuesta del segundo ayudante D. Juan Cesáreo Fernández y Fernández Losada de "una sala destinada a la práctica de operaciones, en dicho Hospital, dotándola de material e instrumental necesario con arreglo a los adelantos de la ciencia" (7). Hasta esta fecha, según Madoz, las operaciones se realizaban en las salas de los pacientes "donde existía un espacio y el material necesario en cada una de ellas para poderlas practicar" (8).

En segundo lugar el intento de creación de varias clínicas especializadas y entre ellas una Clí- 
nica Especial de las Enfermedades de las Vías Urinarias, promovido por el entonces Médico Mayor Enrique Suender en 1877 (Figura 2) (9).

Por último la creación de consultas externas de Urología. Por R.O. del 13 de abril de 1886 quedó aprobado el "Reglamento para las Consultas Externas" para Jefes, Oficiales y sus familiares, en que se hacía referencia a varias especialidades nuevas "según determina la Dirección del Hospital Militar de Madrid". En este reglamento se individualizaban hasta trece disciplinas, algunas sin relación directa con la Medicina Militar. Por separado figuraban: "enfermedades venéreas y sifilíticas" y "afecciones de las vías urinarias y propias de la mujer" (documento existente en Archivo Militar de Segovia citado por Torres) (10). Según Maganto el Hospital Militar fue el segundo Hospital en España en donde se crearon dichas consultas o dispensarios, las primeras fueron las que se abrieron en 1880 con la creación del Instituto Rubio, aunque en este centro las especialidades creadas inicialmente fueron sólo las de Otología y Rinología, Oftalmología y Ginecología (9).

El citado reglamento admite de manera fehaciente, el hecho de que en el Hospital Militar de

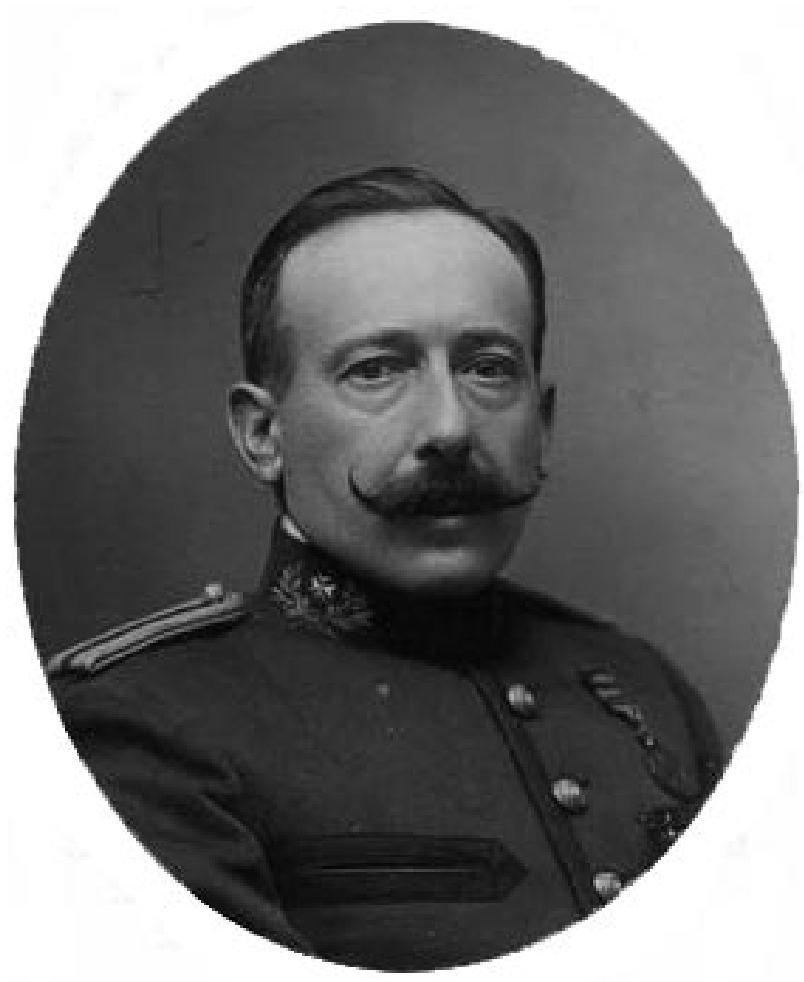

fIGURA 4. Eduardo Mínguez Val, Médico Mayor de Sanidad Militar (1.873 - 1.915).
Madrid se realizaba una Medicina especializada para los pacientes ingresados (11). En su segundo folio Art. 5 se puede leer: "Contando el Hospital militar de esta Plaza con los medios de exploración más modernos por la Ciencia utilizados en el diagnostico y curación de las enfermedades y con Jefes y Oficiales Médicos expertos en el uso y manejo del oftalmoscopio, otoscopio, laringoscopio, plexímetro, cleboscopo, esfigmografo y uretroscopio, así como en la inteligente apreciación de la termometría clínica y análisis físico químico y microscópico de los elementos de los tejidos y de los gérmenes morbíficos cuya proliferación puede ser fecunda en infecciones, dolencias es de trascendental importancia...".

Será en primer lugar el afamado Doctor Enrique Suender florón de la Medicina Militar y pionero de la Urología de Madrid, quien haría una referencia explícita a este padecimiento en su monografía titulada "La Gota Militar" que publicó en 1876 (12) (Figura 3). Según sus propias palabras: "la blenorrea, una afección, por cierto no más frecuente en los militares que en los que ejercen otras profesiones, caracterizada objetivamente por una secreción uretral, purulenta ó moco-purulenta de un líquido turbio, de color amarillento ú opalino, de índole crónica, en ocasiones contagiosa, que unas veces constituye una hipersecrecion continua siempre poco abundante, otras se presenta en forma de filamentos que arrastra el chorro urinario y son visibles en vaso de cristal, y otras se limita a una sola gota, que aglutinando los bordes del meato urinario, opone momentánea resistencia á la emisión de orina".

Según Suender caracterizarían a este padecimiento en primer lugar sus síntomas subjetivos: sensación de calor, cosquilleo, picazón o escozor, limitados a un punto del trayecto de la uretra, asociados a micción dolorosa, tenesmo vesical o rectal y dolores lumbares. Otra característica de la Gota
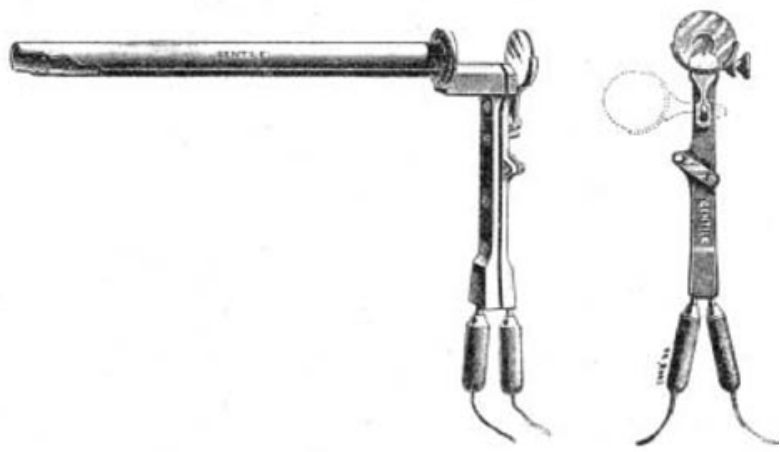

FIGURA 5. Uretroscopio de Luys. 
Militar sería su evolución tórpida: "unos dicen que es la desesperación de los médicos y de los enfermos, y otros la han llamado el oprobio de la cirugía".

Más avanzado su trabajo escribe: "Sin diagnóstico, en una palabra, el tratamiento no puede ser científico, y sus resultados sólo serán felices por una casualidad". Para realizar un diagnóstico correcto sería preciso poseer el habito de manejar varios instrumentos entre los que aparte de la bujía de bola o la metálica de Bell también citará como: "uno de los más importantes el endoscopio de DESORMEAUX ó el uretroscopio de WARVICK..., que permiten ver lo que no es posible apreciar por sensaciones tactiles".

Suender clasificaría las alteraciones capaces de producir la gota militar en:

I.- Estrechez congénita o accidental del meato urinario.

II.- Induración del tejido celular submucoso.

III.- Estrecheces organizadas en cualquier punto del trayecto de la uretra.

IV.- Válvulas uretrales.

V.- Uretritis crónica limitada a la porcion membranosa.

VI.- Granulaciones de la uretra.

VII.- Herpes uretral.

VIII.- Prostatitis sub-aguda.

En su trabajo hace una descripción minuciosa de todas estas patologías y de las consideraciones sobre la base de su experiencia y erudición y al conocimiento de la manera de proceder de su maestro el doctor Reliquet, Montanier y otros autores extranjeros de la época.

En 1879 el bacteriólogo alemán Albert Ludwig Neisser descubriría el germen productor de la gonococia.

Alfredo Rodríguez Viforcos. Médico Segundo del Cuerpo de Sanidad Militar, empleo que no llegó a desempeñar al obtener la Licencia Absoluta aduciendo motivos de salud. Fundó en Madrid la "Revista Especial de Oftalmología, Sifilografía, Dermatología y Afecciones Urinarias", en donde publicaría hasta 17 artículos sobre Dermatología y Urología (13). Mas tarde al crearse el Servicio de Enfermedades del Aparato Genito-urinario en el Hospital Provincial de Madrid $(14,15)$, le sería encargada su jefatura desde su creación en 1889 hasta 1904 en que tras su temprano fallecimiento sería sustituido por $\mathrm{D}$. Antonio Bravo Piqueras que había sido antiguo discípulo de Suender (16).

En La Revista de Sanidad Militar publicada en octubre de 1908, el por entonces Médico
Primero de Sanidad Militar Eduardo Mínguez Val (Figura 4), que ya era conocedor de los procedimientos endoscópicos (17), publicó un articulo con el título: "Uretroscopia y Uretritis", que nos da una autorizada idea de la información, experiencia y rigor que aplicaban en el diagnóstico y tratamiento de esta frecuente enfermedad (18)." Ante el enfermo que acusa síntomas de uretritis..., debe procederse a una exploración metódica que no tiene peligro alguno si se hace con la asepsia necesaria,". Más adelante añade. "pero esta exploración no se ha de considerar acabada si como final de ella no practicamos la uretroscopia".

En el procedimiento para la exploración de la uretra, se procedía en primer lugar a realizar la instilación a través de la uretra de un líquido antiséptico, tras ello se recogía el exudado uretral, en primer lugar, una vez hecho el masaje de las glándulas bulbares y de las de Cowper y en segundo termino de las glándulas de la uretra posterior, de la próstata y de las vesículas seminales. Estos exudados se sometían a estudio histo-bacteriológico. Este proceder nos traslada a los procedimientos modernos para el tratamiento de las prostatitis y las uretritis, popularizados por Meares y Stamey en 1968, que preconizaron la prueba de los cuatro vasos para localizar el asiento de la infección en el tracto urinario inferior. Con lo que se abandonó el masaje prostático terapéutico, al racionalizarse el tratamiento farmacológico.

La exploración física de la uretra se realizaba con el explorador de bola de Guyón que determinaba su calibre y una vez pasado el catéter Benique conveniente se "palpaba" sobre él. Por último para el estudio del estado del epitelio de la mucosa se valían del Uretroscopio de Luys según el proceder del Hospital Necker (Figura 5 y 6 ) (después de haber dejado la uretra dos días en "reposo") y haber procedido a su "lavado" previo.

Minguez Val distinguía la inflamación aguda inespecífica de la inflamación crónica, a su vez dividida en infiltración blanda e infiltración dura. Según sus palabras "como ha demostrado clínicamente el Doctor Motz, de este conocimiento perfecto depende el éxito de la curación". Como método para el tratamiento utiliza lo que llamaba "apósito permanente de Motz", introduciendo en la uretra con la jeringa de pico olivar 4 a 6 c.c. de un medicamento que según la formula de Motz de Paris se componía de Glicerina para reblandecer el epitelio, Hermofenil como antiséptico y Protargol en virtud de la diapedesis que provoca. Se procuraba retener en la uretra dos o tres horas y se repetía cada dos días durante tres o cuatro semanas. 
Son también interesantes las aportaciones que en estos primeros años del siglo XX haría el Médico Mayor Navarro Canovas, sobre el uso de la diatermia en las afecciones urogenitales "Tratamiento de las Orquiepididimitis Gonocócicas mediante la Diatermia" (19); "Tratamiento de la Blenorragia del Hombre y de la Prostatitis Gonocócica mediante la Diatermia" (20) (Figura 7). Con una modificación de las ondas de alta frecuencia de Arsonval que se transforman en calor al aplicarlas a los tejidos del organismo humano. Pensaba, que la elevación de la temperatura en la uretra hasta 42 grados en sus distintos segmentos, hacía desaparecer al gonococo cuya supervivencia es imposible por encima de los 40-4 1 grados. El problema para la uretra quedaba limitado a la construcción de los electrodos adecuados para conseguir el paso de la corriente a través de la misma.

El Dr. Navarro Cánovas diatermiza primero la porción prostática y después la porción anterior de la uretra. Aportaba una experiencia de 30 y 21 casos respectivamente y al parecer con esta técnica original obtenía muy buenos resultados.

No puedo menos que opinar en contra de la falsa novedad en los "actuales" métodos de prostatotermia y termoterapia creados para mejorar la congestión y las molestias irritativas provocados por la Hiperplasia Benigna de la Próstata. Hace ya ochenta años, un Médico Militar español había desarrollado una técnica similar.

Por último, como broche final de este estudio recordaremos al gran urólogo militar español de principios del siglo XX, Rafael Mollá Rodrigo (Médico

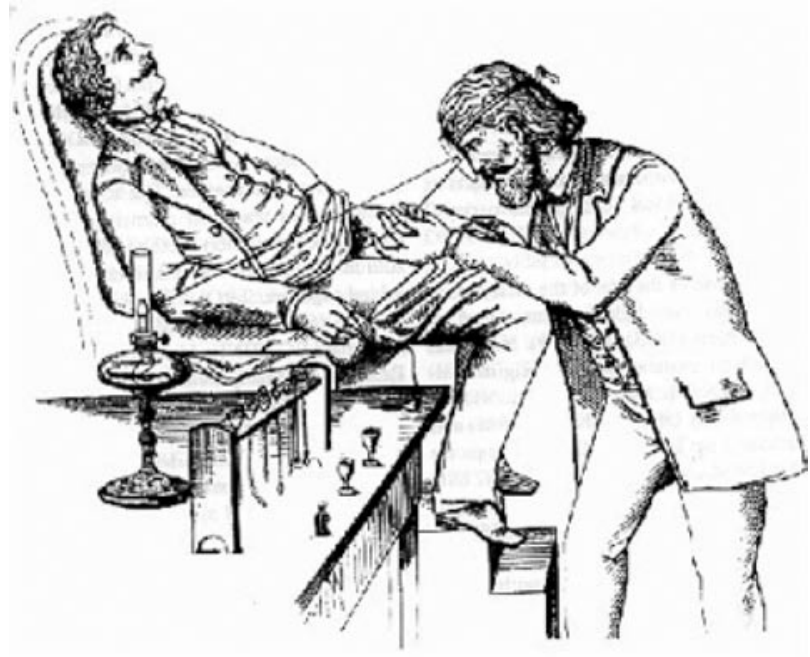

FIGURA 6. Técnica de la Uretroscopia.
Primero) (1862-1930) (Figura 8). Cuya vocación docente y su inclinación por la Cirugía y en especial por la Urología se despertaron precozmente, bajo la enseñanza clínica de los Doctores Suender y Rodríguez Viforcos y asistiendo a las sesiones quirúrgicas de Cirujanos, como el Dr. Rubio, Ribera, Isla, Cervera y Gutiérrez hasta junio de 1892 (21).

Con la muerte de Alejandro San Martín opositó a la cátedra de Cirugía vacante en Madrid, que quedaría desierta por empate entre Mollá y Goyanes. No obstante la obtendría por concurso en 1911 (22). En este mismo año crea en el Sanatorio de Chamartín en Madrid una Cátedra Libre de Urología, al estilo de las que existían en París, en la que imparte la enseñanza de la Especialidad (23).

En 1912 sería elegido Vicepresidente de la Asociación Española de Urología que había sido creada hacía dos años. En 1914 fue nombrado Presidente, cargo que desempeñó durante tres años (24), institucionalizando las Sesiones Científicas que inauguradas el 12 de marzo de 1915, se celebrarían mensualmente hasta el comienzo de la Guerra Civil.

Años antes a su vuelta de Cuba, país en que había sido nombrado Catedrático de Patología Quirúrgica en la Universidad de la Habana, editaría su primer compendio urológico en 1894 titulado: "Resumen Práctico de Diagnóstico y Terapéutica MédicoQuirúrgica de la Afecciones de las Vías Urinarias" (Figura 9). Años más tarde, en 1913 ya como Cate-

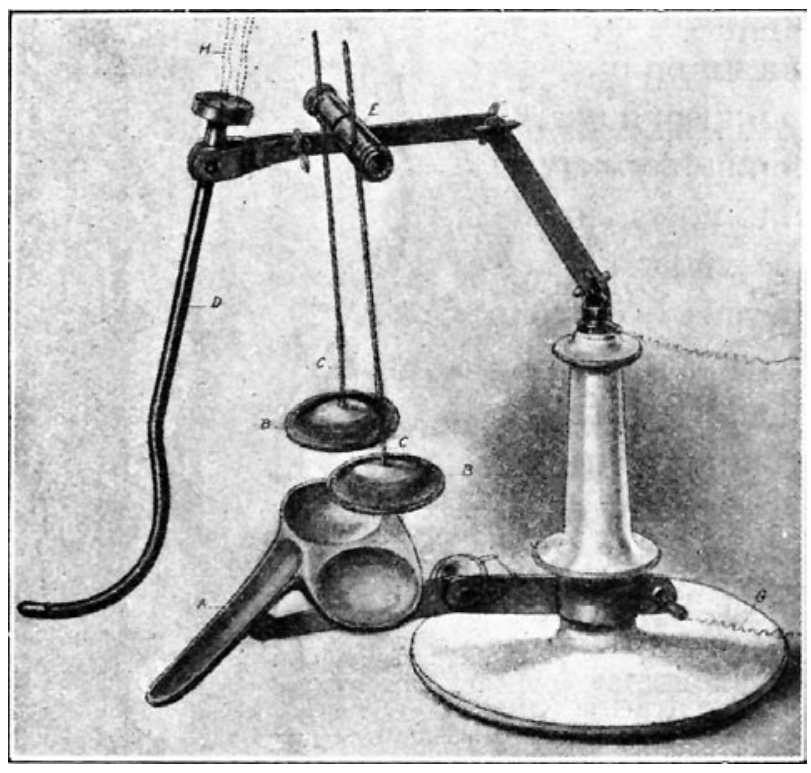

FIGURA 7. Aparato diatérmico de Roucayrol, para actuar sobre la uretra y los testículos. 
drático de Clínica Quirúrgica de Madrid (Miembro de la Asociación Internacional de Urología y Socio fundador y Vicepresidente de la Asociación Española de Urología) editaría su primer libro de texto con importancia docente para la urología española con el título: "Lecciones Clínicas de Urología", que reeditaría muy ampliado en 1921 con el título: "Lecciones Clínicas de Urología y Cirugía Genito-Urinaria". Fruto de su inquietud urológica publicaría más de 40 artículos monográficos sobre distintos temas urológicos (25-27).

Revisando sus libros hemos comprobado la importancia que tenía para los urólogos de la época toda la patología de la uretra, no solo por sus afecciones de tipo infeccioso, ya fuesen agudas que en el caso más frecuente de la uretritis blenorrágica llamaría "blenorragia", o crónicas que denominaría como "blenorrea". Si no también por sus secuelas que en un primer término serían las "estrecheces" y en fases más tardías darían lugar a tumores, abscesos, infiltrados de orina y fístulas.

En sus "Lecciones Clínicas de Urología", dedica seis capítulos a la patología uretral, dando

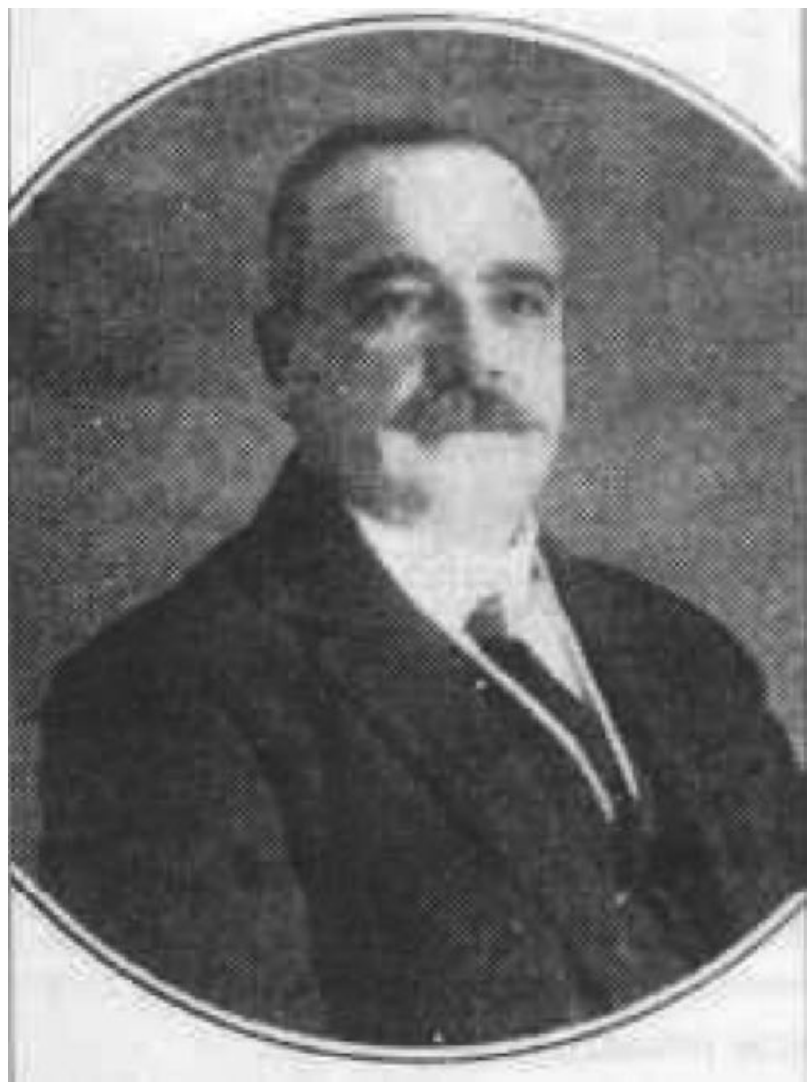

FIGURA 8. El Dr. Rafael Mollá Rodrigo (1862 - 1930). gran importancia tanto al diagnóstico como al tratamiento de los procesos agudos, crónicos y de las complicaciones. Clasifica las uretritis en "Anteriores" y "Posteriores", siendo la demarcación entre ambas el esfínter externo de la uretra.

Hay que reconocer la maestría de aquellos urólogos al interpretar la semiología y en la forma de valorar la exploración física, ya sea mediante la palpación o con la ayuda de instrumentos poco sofisticados, si consideramos que no disponían de técnicas por la imagen, como las que más tarde aportaron la radiología, los contrastes y recientemente la ecografía. También es de admirar su perseverancia al realizar largos tratamientos basados en los lavados antisépticos, ya que no disponían de la antibioterapia, y con las inevitables dilataciones uretrales.

Contemporáneo al Profesor Mollá sería el Dr. D. José Quintana Duque (Coronel Médico) (1869-

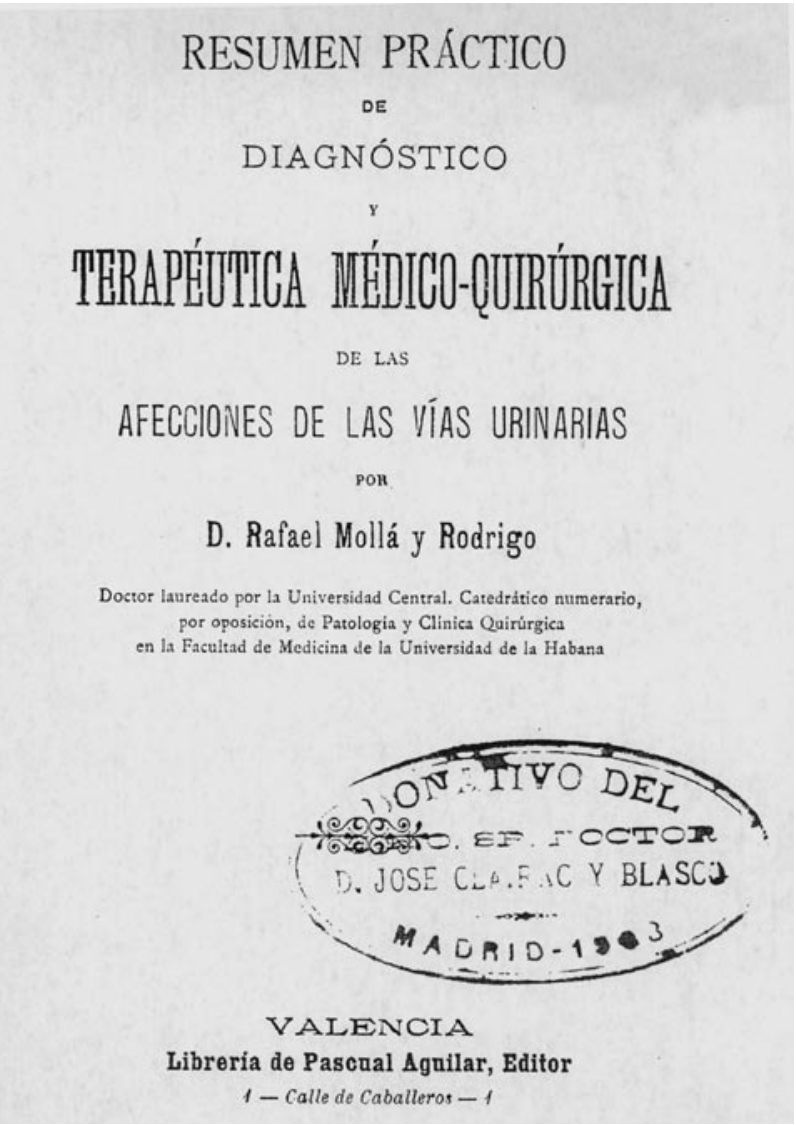

FIGURA 9. "Resumen práctico de Diagnóstico y Terapéutica Médico - Quirúrgica de las Afecciones de las vías urinarias" publicado en 1894 por el profesor Mollá. 


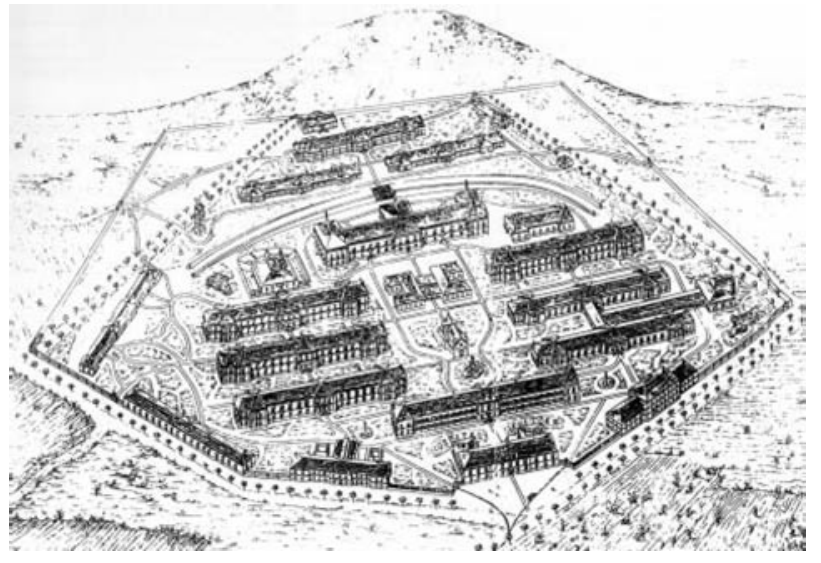

FIGURA 10. Dibujo del Hospital Militar de Carabanchel.

1934). En los más de diez años que permanecería en el Hospital Militar de Carabanchel (Figura 10) desempeñaría diversos cargos. En 1924 sería nombrado Profesor del Curso de Dermo-venereología, sería designado Jefe de Clínica en Sifilografía y Dermatología desde el año 1928 al 1930 así como de las salas $5^{\mathrm{a}}$ y $6^{\mathrm{a}}$ de Venéreo (28).

En la Sanidad Civil el Dr. Quintana fue nombrado en 1921 Vocal del Tribunal para la Oposición a Médicos Adjuntos del Servicio de Profilaxis Publica

\begin{tabular}{|c|c|c|c|}
\hline SUSTANCIAS & & LAVADOS & INYECCIONES \\
\hline \multicolumn{4}{|c|}{ Permanganato Potásico } \\
\hline Idem de zinc & $\zeta$ & $1 \times 4.000$ a $1 \times 8.000$ & $1 \times 500$ a $1 \times 200$ \\
\hline \multicolumn{4}{|l|}{ Idem de plata } \\
\hline Protargol & - & $1 \times 5.000$ & $1 \times 200$ a $1 \times 100$ \\
\hline Argirol & - & 1 a $5 \times 1.000$ & 5 a $10 \times 100$ \\
\hline Choleval & - & $1 \times 5.000$ a $1 \times 1.000$ & $1 / 2 \times 400$ a $1 \times 100$ \\
\hline Albargina & & $>$ & 1 a $2 \times 100$ \\
\hline Phitol & - & 2 a $5 \times 1.000$ & 5 a $10 \times 100$ \\
\hline Colargol & - & $1 \times 1.000$ & $1 \times 100$ \\
\hline \multicolumn{2}{|l|}{ Oxicianuro de mercurio } & $1 \times 4.000$ a $1 \times 6.000$ & $>$ \\
\hline Mercurocromo 220 & - & $1 \times 2.000$ & $0,25 \times 100$ \\
\hline Resorcina & - & $>$ & 1 a $2 \times 100$ \\
\hline Ácido pícrico & - & $>$ & $1 \times 100$ \\
\hline Acriflavina & 1 & & \\
\hline Rivanol & & $1 \times 6.000$ a $1 \times 3.000$ & $1 \mathrm{a} 2 \times 1.000$ \\
\hline Tripaflavina & & & \\
\hline Gonacrina & J & & \\
\hline
\end{tabular}

FIGURA 11. Antisépticos utilizados en los lavados e inyecciones uretrales (Tabla tomada del libro "Prácticas de Urología y Sifilografía" editado por los doctores Pedro Cifuentes y Saínz de Aja). en Madrid de las Enfermedades Venéreo-Sifilíticas. El Dr. Quintana fue un claro representante de aquellos primeros Especialistas que unían conocimientos de distintas ramas de la Medicina. Al mismo tiempo que eran Clínicos expertos en Dermatología y Enfermedades Venéreas, atendían procesos Medico-Quirúrgicos hoy integrados en la Urología, Especialidad con personalidad propia y ya entonces bien diferenciada.

Existe un conocido Tratado editado en España años más tarde en 1927, en que el Dr. Pedro Cifuentes afamado Urólogo y el Dr. Saínz de Aja eminente Dermo-sifilógrafo hacen un estudio muy completo de todas aquellas patologías frecuentes en el periodo que estudia este trabajo (29). A este tratado corresponde una tabla en que se citan los distintos antisépticos que se empleaban para realizar los lavados uretrales (Figura 1 1).

\section{BIBLIOGRAFÍA y LECTURAS RECOMENDADAS (*lectura de interés $y^{* *}$ lectura fundamental)}

1. RIERA, J.: "Historia, Medicina y Sociedad". Pág. 27, Pirámide S.A., Madrid, 1985.

2. LAÍN ENTRALGO, P.: "Historia Universal de la Medicina". Salvat, Barcelona, 1975.

3. RIERA, J.: "Historia, Medicina y Sociedad". Pág. 248-249, Pirámide S.A., Madrid, 1985.

*4. MAGANTO PAVÓN, E.: "Vida y Obra de Enrique Suender (1829-1897) (Patriarca y Alma de la Urología Española)”. Pág.127, Grafinat S.A., Madrid, 1998.

5. VILLALONGA MARTINEZ L.; DOMINGUEZ NIMO, E.: "Vademecum de Sanidad Militar". Pág. 3-7, Romagraf S.A., Madrid, 1987.

6. TORRE FERNANDEZ, J.M.: "Gómez Ulla, Hospital Militar Central, Cien Años de Historia”. Pág. 541-545, Ministerio de Defensa, 1996.

7. DIAZ PRIETO, J.; HIGUES TORIBIO, J.R.: "El primer Hospital Militar de Madrid”. Rev. Ejército; 48: 55.1974.

*8. MADOZ, P.: "Diccionario geográfico-estadístico de España y sus posesiones en Ultramar". Madrid, 1847.

9. MAGANTO PAVÓN, E.: "Historia Biográfica y Bibliográfica de la Urología Española”. Cap. IV. Pág. 207, Edicomplet, Madrid, 2000.

10. TORRE FERNANDEZ, J.M.: "Gómez Ulla, Hospital Militar Central, Cien Años de Historia”. Pág. 45, Ministerio de Defensa, 1996.

11. Reglamento para el servicio de los Hospitales Militares y Ambulancias del Ejército. AGMS: Secc. $2^{\text {a }}$, Div. 14, Legajo 47. 
**12. SUENDER RODRIGUEZ, E.: "La Gota Militar". Madrid, 1876.

13. Expediente personal de Alfredo Rodríguez Viforcos, AGMS, Secc. $1^{\text {a }}$, Div. $1^{\text {a }}$, Legajo R-1575.

14. PULIDO FÉRNANDEZ, A.: "Memoria sobre reformas de los Servicios de los Hospitales Provinciales de Madrid". Madrid, 1889.

15. ALVAREZ SIERRA, J.: "Diccionario de Autoridades médicas". Editora Nacional, Madrid, 1963.

16. MAGANTO PAVÓN, E.: "Vida y Obra de Enrique Suender (1829-1897) (Patriarca y Alma de la Urología Española)”. Grafinat S.A., Madrid, Pág. 62, 1998.

17. MÍNGUEZ VAL, E.: "Lavado de la pelvis del riñón". Rev. De Sanidad Militar, 5: 97, 1908.

**18. MÍNGUEZ VAL, E.: "Uretroscopia y uretritis". Rev. De Sanidad Militar, 20: 517, 1908.

*19. NAVARRO CANOVAS, B.: "Tratamiento de la orquiepididimitis gonocócicas mediante diatermia”. Rev. De Sanidad Militar, 3: 64, 1916.

*20. NAVARRO CANOVAS, B.: "Tratamiento de la blenorragia del hombre y de la prostatitis gonocócica mediante la diatermia". Rev. De Sanidad Militar, 16: 505, 1918.
*21. PÉREZ ALBACETE, M.: "Dr. D. Rafael Mollá y Rodrigo (1862-1930)". Urol. Integr. Invest., 7: 166, 2002.

22. MAGANTO PAVÓN, E.: "Historia Biográfica y Bibliográfica de la Urología Española”. Pág. 242243, Edicomplet, Madrid, 2000.

23. PÉREZ ALBACETE, M.: "Dr. D. Rafael Mollá y Rodrigo (1862-1930)". Urol. Integr. Invest., 7: 166, 2002.

24. Expediente personal de Rafael Mollá Rodrigo, AGMS, Secc.1 ${ }^{\text {a }}$, Div. $1^{\text {a }}$, Legajo M-3621.

**25. MOLLÁ RODRIGO, R.: "Resumen Práctico de Diagnóstico y Terapéutica Médico-Quirúrgica de las Afecciones de las Vías Urinarias". Pág. 67146, Pascual Aguilar, Valencia, 1894.

26. MOLLÁ RODRIGO, R.: "Lecciones Clínicas de Urología”. Pág.133-260. Casa Vidal, Madrid, 1913.

27. MOLLÁ RODRIGO, R.: "Lecciones Clínicas de Urología y Cirugía Génito-Urinaria”. Julio Cosano, Madrid, 1921.

28. Expediente personal de José Quintana Duque, AGMS, Secc.1 $1^{\text {a }}$, Div. 1 ${ }^{\mathrm{a}}$, Legajo Q-120.

29. CIFUENTES DIAZ, P.; SAIINZ AJA, E.A.: "Prácticas de Urología y Sifilografía". Javier Morata, Madrid, 1927. 